\title{
The Genetics Corner: A Consultation for Neonatal Diabetes Mellitus Reveals Uniparental Disomy 6
}

Subhadra Ramanathan MS, MSc, Matthew Wood MD, Robin Dawn Clark MD

\section{Case History:}

A small for gestational age female infant was transferred to the NICU at Loma Linda University Children's Hospital at seven days of age for persistent hyperglycemia and neonatal diabetes mellitus. Persistent maternal vaginal bleeding was reported from 2 months' gestation. The mother reported nausea and hyperemesis throughout the pregnancy and a poor gestational weight gain of 11 lbs. Intra-uterine growth retardation (IUGR) was recognized late in the third trimester.

\section{"The mother reported nausea and hyperemesis throughout the pregnancy and a poor gestational weight gain of 11 lbs. Intra-uterine growth retardation (IUGR) was recognized late in the third trimester."}

The baby was delivered by induced vaginal delivery at $37 \mathrm{w} 3 \mathrm{~d}$ for IUGR to a 28-year-old G6 P2-3 SAb1 TAb2 mother.

Birth weight:

Birth length:

Birth head circumference $4 \mathrm{lb} 11.1 \mathrm{oz}(2130 \mathrm{~g})\left(3^{\text {rd }}\right.$ percentile $)$ $17.5^{\prime \prime}(44.5 \mathrm{~cm})\left(8^{\text {th }}\right.$ percentile $)$

After delivery at an outside hospital, blood glucose values were initially stable in the $70 \mathrm{~s}$, but repeat checks showed elevated blood sugars trending up above 200. A continuous insulin infusion did not control the hyperglycemia. Blood glucose values ranged from $>500$ to $<70$ in spite of frequent adjustments to insulin infusion. C-peptide was undetectable while on an insulin infusion. The patient was transferred to our facility for endocrinology consult and further management.

Under endocrinology direction, insulin infusion therapy transitioned to oral glyburide. After discontinuation of insulin infusion and prior to initiation of glyburide, the C-peptide level was detectable but low, in spite of rising blood sugar levels. There was no evidence of multiple common diabetes-related autoantibodies. Due to elevated blood sugars, glyburide dosing was increased, and subcutaneous insulin glargine was started. While on both insulin glargine and glyburide, blood sugars remained labile, with both high and low blood sugar values. Insulin glargine was discontinued, and glyburide was increased further under endocrinology direction. At this time, blood glucose is more stable but elevated at $100-300$.

\section{Genetics Evaluation:}

Because of restrictions due to the coronavirus pandemic, this evaluation was done at a distance. The NICU team uploaded photos of the baby into the Media tab of her electronic medical record. Examination of these photographs revealed a thin infant with deep infraorbital creases, macroglossia with a long, protruding tongue, mild micrognathia, thin upper lip, and incomplete helical folds of the right external ear. She had redundant periumbilical skin, which gave the impression that a small umbilical hernia had recently been reduced. She had asymmetric growth retardation with decreased subcutaneous tissue, wrinkled skin, and a relatively preserved head circumference. An echocardiogram was normal; an ultrasound of the abdomen showed normal pancreas.

The clinical history and physical findings suggested transient neonatal diabetes mellitus caused by paternal uniparental disomy for chromosome 6 (patUPD6). Chromosome microarray analysis was

\section{"The clinical history and physical findings suggested transient neonatal diabetes mellitus caused by paternal uniparental disomy for chromosome 6 (patUPD6)."}

pending at that time, and requests for other genetic tests were put on hold until the microarray results were available. In a few days, the microarray results confirmed uniparental isodisomy 6 : $\operatorname{arr}(\mathrm{X}) \times 2,(6) \times 2 \mathrm{hmz}$. Methylation studies were not done, but the phenotype was consistent with UPD6 of paternal origin. No further genetic testing was ordered.

\section{Discussion and Counseling:}

The diagnosis of transient neonatal diabetes mellitus due to paternal uniparental disomy 6 (6q24-TNDM) explains this child's low birth weight and macroglossia as well as her neonatal diabetes. The cardinal features of 6q24-TNDM are severe intrauterine growth retardation, neonatal hyperglycemia, which usually starts in the first week of life in a term infant, dehydration, and absence of ketoacidosis. The low birth weight is likely caused by the lack of insulin in utero. Insulin is a major growth factor during fetal life. Macroglossia and umbilical hernia are common. Our patient's clinical presentation and chromosome microarray test results are consistent with 6q24-TNDM due to paternal isodisomy for chromosome 6.

The "transient" neonatal diabetes mellitus associated with 6q24TNDM lasts three months on average but can persist until 18 months. Insulin is usually required initially, but the need for it gradually declines over time. Intermittent episodes of hyperglycemia may occur in childhood, particularly during illnesses, during which the child should be closely monitored. Diabetes mellitus may recur in adolescence or later in adulthood. Intelligence and growth 
are usually normal. However, the long-term outcomes are still not completely known

Neonatal or congenital diabetes mellitus (NDM) is rare, with an incidence of about $1 / 90,000-160,000$ live births. There are over 20 known causes for NDM, which can be classified into transient, permanent, and syndromic forms. In a recently published study from the United Kingdom involving 1020 patients, genetic testing detected a causal genetic etiology in more than $80 \%$ of infants diagnosed with diabetes before six months of age (de Franco $E$ et al., 2015). Mutations in the potassium channel genes are the most common cause of permanent neonatal diabetes mellitus.

A genetic aberration usually causes transient neonatal diabetes mellitus at the imprinted locus on chromosome 6q24 (6q24TNDM). Genomic imprinting refers to the differential expression of genes based on parent-of-origin, usually mediated by DNA methylation, which silences gene expression in one of the alleles.

Three distinct mechanisms cause 6q24-TNDM by overexpression of the imprinted genes PLAGL1 and HYMAI at chr6q24, which are normally expressed only on the paternally derived chromosome 6

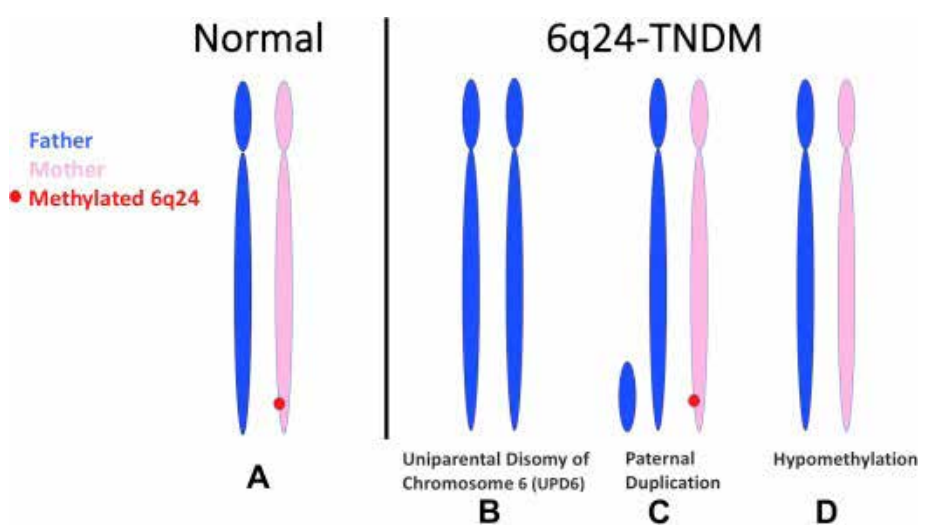

Figure 1 from Lemelman MB, Letourneau L, Greely SAW, 2018).

A- Normal biparental disomy of chromosome 6: active genes are expressed on the paternal copy of chromosome 6 , silenced (imprinted) genes are not expressed on the maternal copy of chromosome 6

B- Uniparental disomy of chromosome 6: both copies of chromosome 6 are derived from the father, which produces two active copies of these genes without a maternally derived copy of chromosome 6 .

C- Duplication of the paternal allele of the imprinted region on chr 6q24: two active copies of these genes on the paternal chromosomes, one inactive (imprinted) copy on the maternally derived copy.

D- Loss of maternal methylation (hypomethylation) of the imprinted region on chr 6q24: the maternally derived copy is

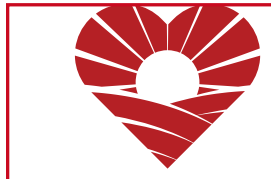

CONGENITAL CARDIOLOGY
The only worldwide monthly publication exclusively serving Pediatric and Adult Cardiologists that focus on Congenital/ Structural Heart Disease (CHD), and Cardiothoracic Surgeons. active rather than silenced.

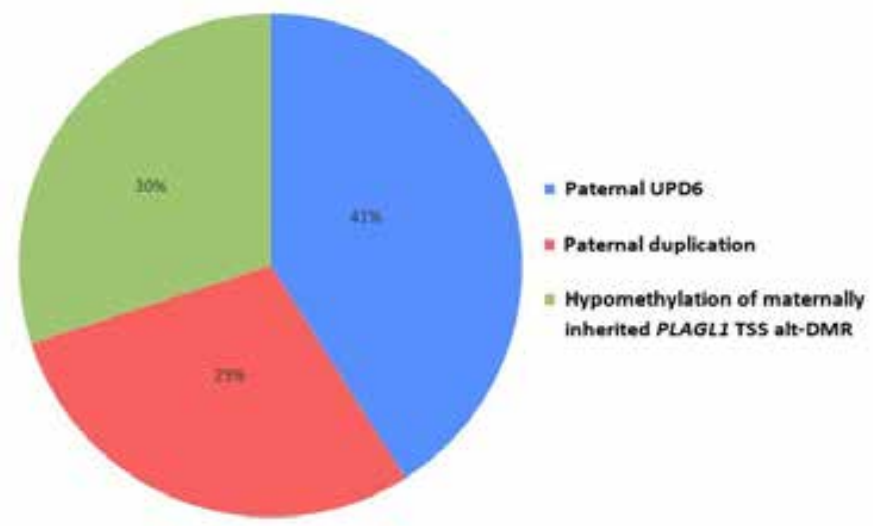

Figure 2: Distribution of the different genetic mechanisms causing 6q24-TNDM, from GeneReviews on 6q24-TNDM

Chromosome microarray analysis using SNP (single nucleotide polymorphism) genotyping can detect uniparental isodisomy as well as a duplication of $6 q 24$.

The microarray test detected isodisomy of chromosome 6 in our patient. The likely mechanism was "monosomy rescue," in which monosomy for chr 6 in the egg was compensated for by post-zygotic mitotic duplication of the paternal chr 6 , leading to complete isodisomy of the paternal chr 6 . Monosomy 6 is not compatible with life, but after diploidy has been restored, the embryo can continue to develop. Therefore, paternal UPD "rescues" an embryo that would not have been viable otherwise (Figure 3).

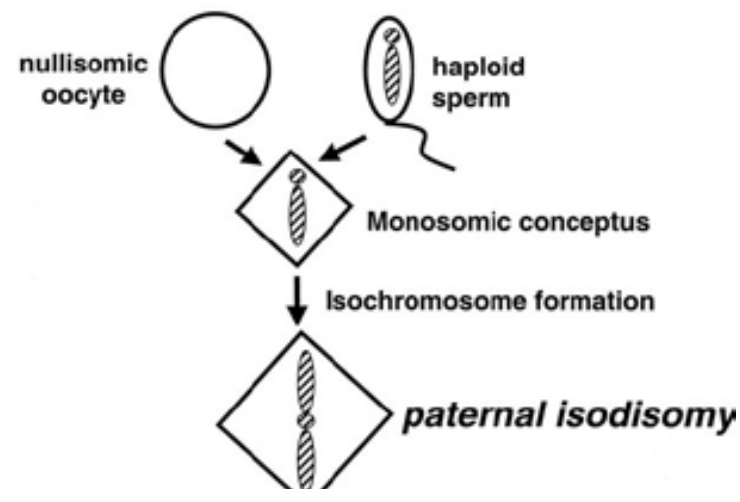

Figure 3: Monosomy rescue through isochromosome formation (adapted from Shaffer LG et al., 2001)

Practical applications:

1. Consider 6q24-TNDM when a small for gestational age infant presents with macroglossia and neonatal diabetes.

2. Recognize that the "transient" nature of TNDM, may still take
Subscribe Electronically Free on the Home Page www.CongenitalCardiologyToday.com 
months to resolve.

3. A chromosome microarray can identify most cases of 6q24TNDM. However, methylation studies of chromosome 6, which may require parental blood samples, can identify all three major causes.

4. Genetic testing informs the treatment of the various subtypes of NDM. A clear etiology and diagnosis clarifies the natural history, prognosis, and risks for developing related disorders.

References:

1. De Franco E, Flanagan SE, Houghton JA, et al. The effect of early, comprehensive genomic testing on clinical care in neonatal diabetes: an international cohort study. Lancet. 2015;386(9997):957:963

2. Lemelman MB, Letourneau L, Greeley SAW. Neonatal Diabetes Mellitus: An Update on Diagnosis and Management. Clin Perinatol. 2018;45(1):41:59

3. Temple IK, Mackay DJG. Diabetes Mellitus, 6q24-Related Transient Neonatal. 2005 Oct 10 [Updated 2018 Sep 13]. In: Adam MP, Ardinger HH, Pagon RA, et al., editors. GeneReviews ${ }^{\circledR}$ [Internet]. Seattle (WA): University of Washington, Seattle; 1993-2020. Available from: https://www.ncbi.nlm. nih.gov/books/NBK1534/

4. Shaffer LG, Agan N, Goldberg JD, Ledbetter DH, Longshore JW, Cassidy SB. American College of Medical Genetics statement of diagnostic testing for uniparental disomy. Genet Med. 2001;3(3):206:211

The authors have no relevant disclosures.

NT
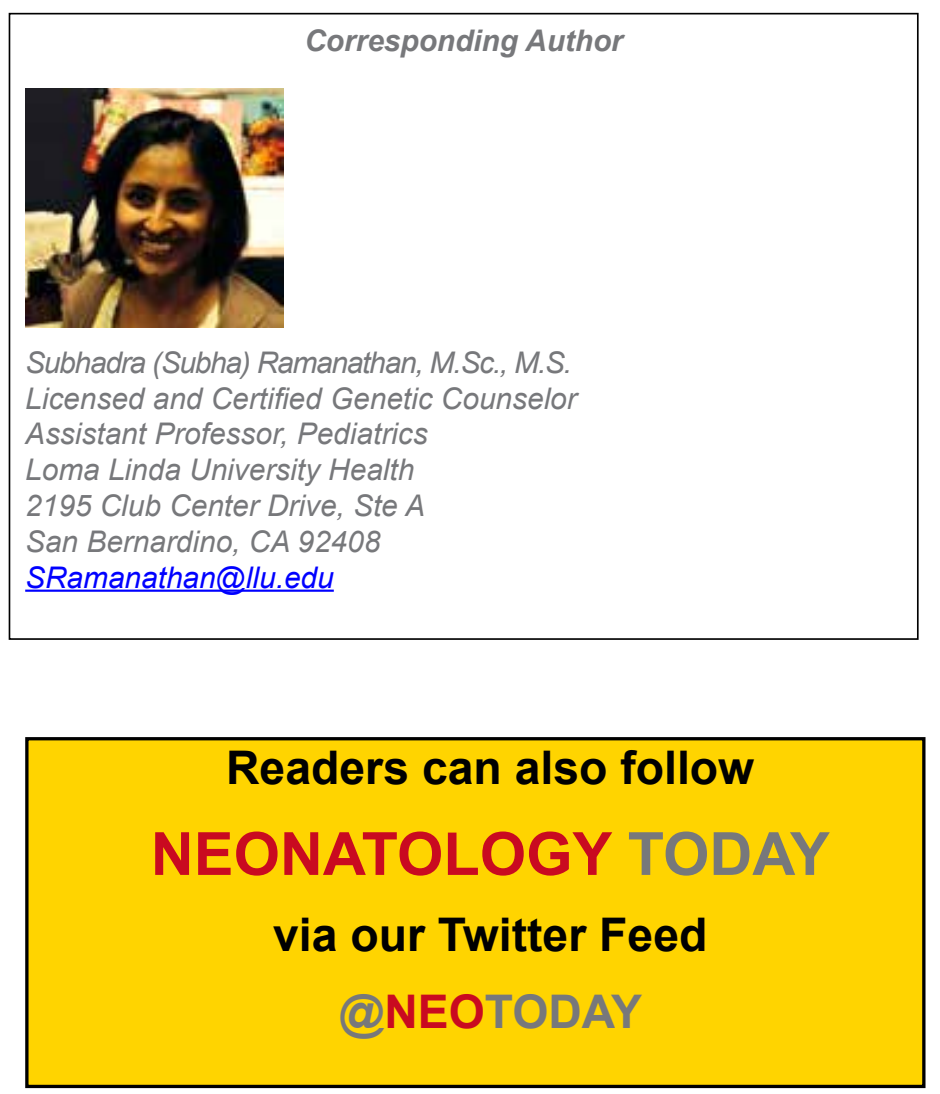
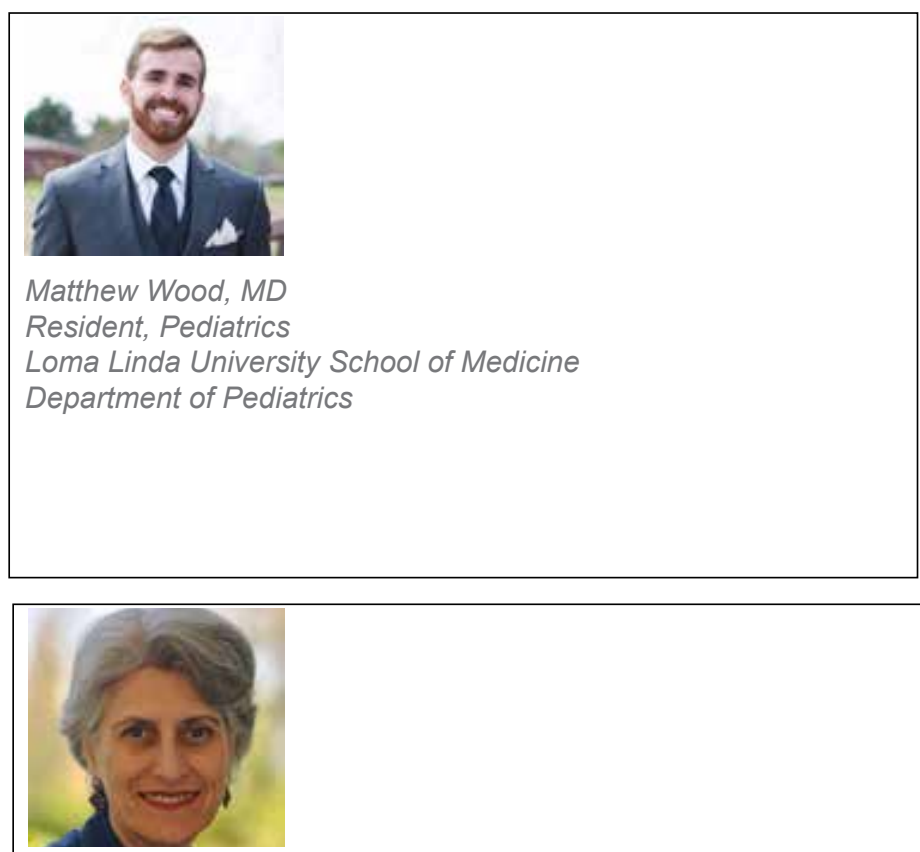

Robin Clark, MD

Professor, Pediatrics

Loma Linda University School of Medicine

Division of Genetics

Department of Pediatrics

rclark@/lu.edu

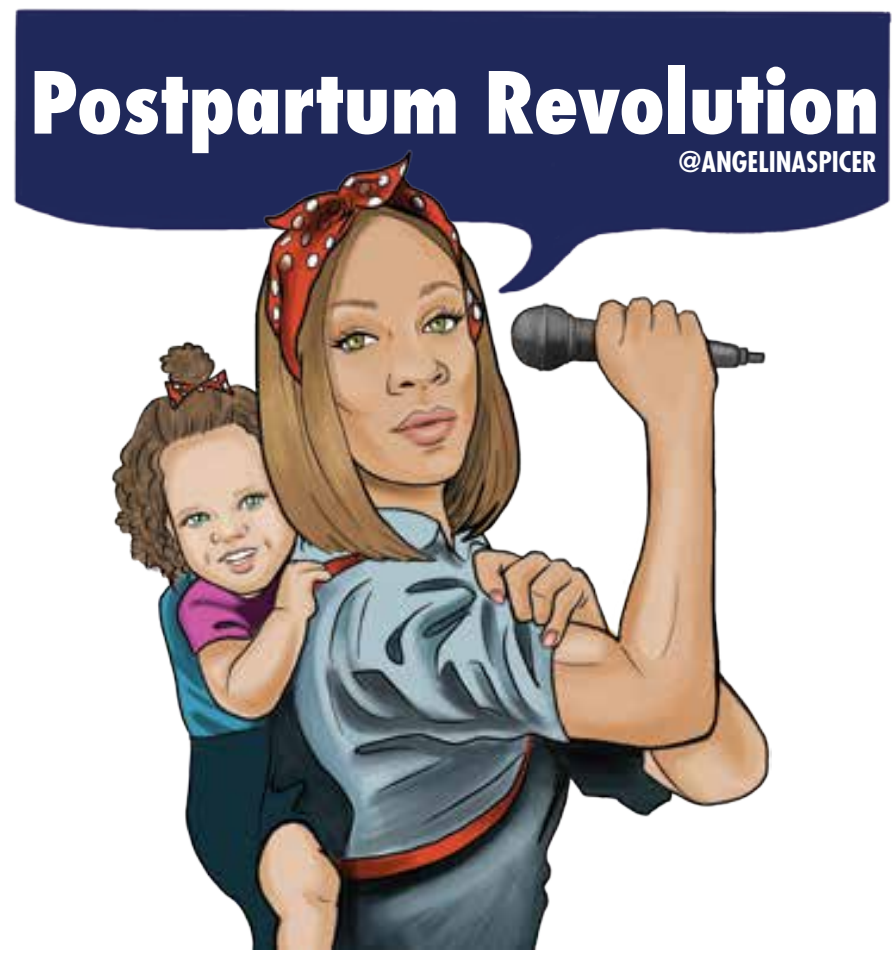

